\title{
Terminal Sliding Mode Control for Attitude Tracking of Spacecraft Based on Rotation Matrix
}

\author{
Yong Guo, Shen-Min Song, and Xue-Hui Li \\ Center for Control Theory and Guidance Technology, Harbin Institute of Technology, Harbin 150001, China \\ Correspondence should be addressed to Shen-Min Song; songshenmin@hit.edu.cn
}

Received 6 June 2014; Revised 14 August 2014; Accepted 1 September 2014

Academic Editor: Sabri Arik

Copyright (c) 2015 Yong Guo et al. This is an open access article distributed under the Creative Commons Attribution License, which permits unrestricted use, distribution, and reproduction in any medium, provided the original work is properly cited.

\begin{abstract}
Two finite-time controllers without unwinding for the attitude tracking control of the spacecraft are investigated based on the rotation matrix, in which a novel modified nonsingular fast terminal sliding manifold is developed to $\operatorname{keep} \operatorname{tr}(\widetilde{\mathbf{R}}) \neq-1$. The first terminal sliding mode controller can compensate external disturbances with known bounds, while the second one can compensate external disturbances with unknown bounds by using an adaptive control method. Since the first terminal sliding mode controller is continuous, it is able to avoid chattering phenomenon. Theoretical analysis shows that both the two controllers can make spacecraft follow a time-varying reference attitude signal in finite time. Numerical simulations also demonstrate that the proposed control schemes are effective.
\end{abstract}

\section{Introduction}

Because of the important applications in many space missions such as in-orbit maintenance and space station installation, the attitude control of the spacecraft has gained extensive interests in recent years. Many researchers have developed various controllers for them based on the unit quaternion [ 1 , 2], dual quaternion [3], and modified Rodrigues parameters (MRP) $[4,5]$. However, those representations are unable to represent the set of attitudes both globally and uniquely, so that the controllers usually result in unwinding [6], which means that it will cost extra fuel consumption by the spacecraft traveling a large distance before returning to the desired attitude when the closed-loop system is close to the desired attitude equilibrium.

In order to avoid unwinding, many controllers based on the rotation matrix have been developed [7-10]. Weiss et al. [7] derived two controllers for spacecraft attitude tracking problems without unwinding. Sanyal et al. [8] gave a continuous controller for spacecraft attitude tracking of arbitrary continuously differentiable attitude trajectories. Chaturvedi et al. [9] presented a complete analysis for two problems on the stabilization of the inverted 3D pendulum. Because controllers [7-9] adopted the attitude error function $e=$ $0.5 \operatorname{tr}(\mathbf{I}-\widetilde{\mathbf{R}})$ which was not proportional to the rotation angle, the performance of those controllers became worse when the initial error became larger. To deal with this problem, Lee [10] proposed an attitude controller by a new attitude error function for the tracking control system on $\mathrm{SO}(3)$. Since the aforementioned controllers provided asymptotic stability [79] and exponential stability [10], the systems could converge to the equilibrium only when the time goes to infinity. Thus, the finite-time stabilization implying better performance is attracting more and more attention.

Terminal sliding mode (TSM) control is one of finitetime control schemes that provide faster convergence speed. However, TSM control has three disadvantages that are singularity, chattering, and slower convergence speed when the system state is far away from the equilibrium. Therefore, many improved TSM algorithms have been developed. Feng et al. [11] gave a nonsingular TSM controller for a secondorder system to eliminate the singularity problem. Yu and Zhihong [12] designed a fast TSM controller combining advantages of the traditional linear hyperplane-based sliding mode control and TSM control to improve the convergence speed. The discontinuous controllers [11, 12] can lead to 
chattering which may arouse high-frequency unmodeled plant dynamics. In general, the boundary layer approach is used to eliminate the chattering; however, the finite-time stability will be lost in the boundary layer. Yu et al. [13] proposed a continuous finite-time controller that can enjoy benefits of both high precision and chattering attenuation by properly choosing the fractional powers.

TSM control has led wide applications in attitude tracking of the spacecraft. A number of research works have been reported on this topic in the past decades. Wu et al. [14] investigated two robust sliding mode controllers based on the quaternion and Lagrange-like model to solve the spacecraft attitude tracking control problem. Lu and Xia [15] investigated the attitude tracking control problem for the rigid spacecraft under input saturation with finite-time stabilization. Zou and Kumar [16] proposed a distributed attitude coordination control scheme using TSM control for a group of spacecraft in the presence of external disturbances. Pukdeboon [17] proposed two second-order sliding mode controllers to solve the attitude tracking control of a spacecraft with external disturbances and inertia uncertain.

To the best knowledge of the authors, there are rarely approaches can provide finite-time control without unwinding for a spacecraft except our previous article [18]. In our previous article, we investigated two finite-time controllers for the attitude control of the spacecraft by an adaptive backstepping method. Because the two controllers were designed in the region of attraction, it needs to be further studied.

To overcome these drawbacks, we investigate the finitetime control by using TSM control. Compared with the listed literatures, the contributions are summarized as follows. (i) A novel modified nonsingular fast terminal sliding manifold is developed to keep $\operatorname{tr}(\widetilde{\mathbf{R}}) \neq-1$. (ii) Two controllers without unwinding are proposed for the attitude control of the spacecraft by using TSM control. (iii) Compared with the controllers in [18], the two controllers are almost globally finite-time stabilization.

This paper is organized as follows. An attitude dynamic model is established in the following section. In Section 3, a state error is given. Then, two controllers are proposed. Furthermore, the corresponding stability proofs are given as well. Numerical simulations are presented in Section 4. The paper is closed with some concluding remarks.

\section{Spacecraft Attitude Dynamics}

The spacecraft is modeled as rigid body based on the rotation matrix. Specifically, the equation of motion of the spacecraft is defined as (1) and (2). Here, $\mathbf{R}$ is the rotation matrix that transforms the body frame into the inertial frame resolved in the body frame. $\omega \in R^{3 \times 1}$ is the angular velocity in the body frame. $\mathbf{d} \in R^{3 \times 1}$ and $\mathbf{u} \in R^{3 \times 1}$ are the external disturbance torque and control torque, respectively. $\mathbf{J} \in R^{3 \times 3}$ is the inertia matrix. Consider

$$
\begin{gathered}
\dot{\mathbf{R}}=\mathbf{R} \omega^{\times}, \\
\mathrm{J} \dot{\omega}=-\omega^{\times} \mathrm{J} \omega+\mathbf{u}+\mathbf{d},
\end{gathered}
$$

$$
\boldsymbol{\omega}^{\times}=\left[\begin{array}{ccc}
0 & -\omega_{3} & \omega_{2} \\
\omega_{3} & 0 & -\omega_{1} \\
-\omega_{2} & \omega_{1} & 0
\end{array}\right] .
$$

$\mathbf{R}_{d}$ and $\boldsymbol{\omega}_{d} \in R^{3 \times 1}$ are the reference attitude and angular velocity in the reference frame, respectively. $\widetilde{\mathbf{R}}=\mathbf{R}_{d}^{T} \mathbf{R}$ and $\widetilde{\boldsymbol{\omega}}=\boldsymbol{\omega}-\widetilde{\mathbf{R}}^{T} \boldsymbol{\omega}_{d}$ are the rotation matrix error and angular velocity error in the body frame. Because $\widetilde{\mathbf{R}}$ is a matrix, it cannot be used to design the controller directly. A new attitude error is constructed in [10] which is defined as (4). The map $\vee$ transforms a skew-symmetric matrix to a vector. For example $\left(\mathbf{a}^{\times}\right)^{\vee}=\mathbf{a}$ and $\left(\mathbf{A}^{\vee}\right)^{\times}=\mathbf{A}$, where $\mathbf{a} \in R^{3 \times 1}$ and $\mathbf{A}$ is a skew-symmetric matrix. Consider

$$
\mathbf{e}_{\widetilde{\mathbf{R}}}=\frac{1}{2 \sqrt{1+\operatorname{tr}(\widetilde{\mathbf{R}})}}\left(\widetilde{\mathbf{R}}-\widetilde{\mathbf{R}}^{T}\right)^{\vee} .
$$

In combination with (1)-(4), the equation of motion of the spacecraft is given by the following equations:

$$
\begin{gathered}
\dot{\mathbf{e}}_{\widetilde{\mathbf{R}}}=\mathbf{E} \widetilde{\boldsymbol{\omega}}, \\
\mathbf{J} \dot{\tilde{\boldsymbol{\omega}}}=\mathbf{F}+\mathbf{u}+\mathbf{d}, \\
\mathbf{F}=-\left(\widetilde{\boldsymbol{\omega}}+\widetilde{\mathbf{R}}^{T} \boldsymbol{\omega}_{d}\right)^{\times} \mathbf{J}\left(\widetilde{\boldsymbol{\omega}}+\widetilde{\mathbf{R}}^{T} \boldsymbol{\omega}_{d}\right)+\mathbf{J} \widetilde{\boldsymbol{\omega}}^{\times} \widetilde{\mathbf{R}}^{T} \boldsymbol{\omega}_{d}-\widetilde{\mathbf{J}}^{T} \dot{\boldsymbol{\omega}}_{d}, \\
\mathbf{E}=\frac{1}{2 \sqrt{1+\operatorname{tr}(\widetilde{\mathbf{R}})}}\left(\operatorname{tr}(\widetilde{\mathbf{R}}) \mathbf{I}-\widetilde{\mathbf{R}}^{T}+2 \mathbf{e}_{\widetilde{\mathbf{R}}} \mathbf{e}_{\widetilde{\mathbf{R}}}^{T}\right) .
\end{gathered}
$$

\section{Design of the Controller}

We employ the idea of the finite-time control to design the robust controllers for the attitude control of the spacecraft by using TSM control. The following lemmas are useful to design the finite-time controllers.

Lemma 1 (see [10]). Let $\mathbf{R}_{d}^{T} \mathbf{R}=\exp \left(\mathbf{x}^{\times}\right)$, and there exists $\mathbf{x} \in R^{3}$ with $\|\mathbf{x}\| \leq \pi$. The matrix 2-norm of $\mathbf{E}$ is $\|\mathbf{E}\|=1 / 2$. Furthermore, if $\|\mathbf{x}\| \neq \pi, \mathbf{E}$ is an invertible matrix, $\left\|\mathbf{e}_{\widetilde{\mathbf{R}}}\right\|<1$, and $-1<\operatorname{tr}(\widetilde{\mathbf{R}}) \leq 3$.

Lemma 2 (see [13]). Supposing that $\alpha_{1}, \alpha_{2}, \ldots, \alpha_{n}$ are all positive numbers and $0<\rho<2$, then the following inequality holds:

$$
\left(\alpha_{1}^{2}+\cdots+\alpha_{n}^{2}\right)^{\rho} \leq\left(\alpha_{1}^{\rho}+\cdots+\alpha_{n}^{\rho}\right)^{2} .
$$

Lemma 3 (see [13]). Supposing that $\dot{V}(t) \leq-\alpha V(t)-\beta V(t)^{\gamma}$, $\forall t_{1} \geq t_{0}$, where $\alpha>0, \beta>0,0<\gamma<1$, and $V(t)$ is a continuous positive definite function, then the system converges to the equilibrium point in finite time:

$$
t_{1} \leq t_{0}+\frac{1}{\alpha(1-\gamma)} \ln \frac{\alpha V\left(t_{0}\right)^{1-\gamma}+\beta}{\beta} .
$$

Assumption 4. d, $\boldsymbol{\omega}_{d}$, and $\dot{\boldsymbol{\omega}}_{d}$ are assumed to satisfy $\|\mathbf{d}\| \leq$ $d_{\max }$ and $\left\|\omega_{d}+\dot{\boldsymbol{\omega}}_{d}\right\| \leq \omega_{d \max }$, respectively. Here, $d_{\max }$ and $\omega_{d \max }$ are positive constants. 
In order to keep $\left\|\mathbf{e}_{\widetilde{\mathbf{R}}}\right\|<1$, we design the fast TSM as (11), where $\alpha, \beta, \lambda$, and $\eta$ are positive constants, $0<$ $\gamma<1, r_{1}=(2-\gamma) \eta^{\gamma-1}, r_{2}=(\gamma-1) \eta^{\gamma-2}, \mathbf{f}\left(\mathbf{e}_{\widetilde{\mathbf{R}}}\right)=$ $\left[f\left(\mathbf{e}_{\widetilde{\mathbf{R}}, 1}\right), f\left(\mathbf{e}_{\widetilde{\mathbf{R}}, 2}\right), f\left(\mathbf{e}_{\widetilde{\mathbf{R}}, 3}\right)\right]^{T}$, and $\operatorname{sig}\left(\mathbf{e}_{\widetilde{\mathbf{R}}, i}\right)^{\gamma}=\left|\mathbf{e}_{\widetilde{\mathbf{R}}, i}\right|^{\gamma} \operatorname{sign}\left(\mathbf{e}_{\widetilde{\mathbf{R}}, i}\right)$. Consider

$$
\begin{aligned}
\mathbf{S} & =\dot{\mathbf{e}}_{\widetilde{\mathbf{R}}}+\alpha \mathbf{e}_{\widetilde{\mathbf{R}}}+\beta \mathbf{f}\left(\mathbf{e}_{\widetilde{\mathbf{R}}}\right)+\lambda\left(\ln \frac{1}{1-\mathbf{e}_{\widetilde{\mathbf{R}}}^{T} \mathbf{e}_{\widetilde{\mathbf{R}}}}\right) \mathbf{e}_{\widetilde{\mathbf{R}}}, \\
f\left(\mathbf{e}_{\widetilde{\mathbf{R}}, i}\right) & = \begin{cases}r_{1} \mathbf{e}_{\widetilde{\mathbf{R}}, i}+r_{2} \operatorname{sign}\left(\mathbf{e}_{\widetilde{\mathbf{R}}, i}\right) \mathbf{e}_{\widetilde{\mathbf{R}}, i}^{2} & \left|\mathbf{e}_{\widetilde{\mathbf{R}}, i}\right| \leq \eta, i=1,2,3 \\
\operatorname{sig}\left(\mathbf{e}_{\widetilde{\mathbf{R}}, i}\right)^{\gamma} & \text { others. }\end{cases}
\end{aligned}
$$

Based on the TSM control, the control law for the spacecraft is given by the following equation:

$$
\begin{aligned}
\mathbf{u}=- & \mathbf{J} \mathbf{E}^{-1}\left(\dot{\mathbf{E}} \widetilde{\boldsymbol{\omega}}+\alpha \dot{\mathbf{e}}_{\widetilde{\mathbf{R}}}+\frac{2 \lambda \mathbf{e}_{\widetilde{\mathbf{R}}}^{T} \dot{\mathbf{e}}_{\widetilde{\mathbf{R}}}}{1-\mathbf{e}_{\widetilde{\mathbf{R}}}^{T} \mathbf{e}_{\widetilde{\mathbf{R}}}} \mathbf{e}_{\widetilde{\mathbf{R}}}+\lambda\left(\ln \frac{1}{1-\mathbf{e}_{\widetilde{\mathbf{R}}}^{T} \mathbf{e}_{\widetilde{\mathbf{R}}}}\right) \dot{\mathbf{e}}_{\widetilde{\mathbf{R}}}\right. \\
& \left.+\beta \dot{\mathbf{f}}\left(\mathbf{e}_{\widetilde{\mathbf{R}}}\right)+k_{1} \mathbf{S}+k_{2} \operatorname{sig}(\mathbf{S})^{\gamma}\right)-\mathbf{F}
\end{aligned}
$$

Theorem 5. Consider a spacecraft described by (5)-(6) under Assumption 4 where $d_{\max }$ is a given positive constant. By applying the proposed control scheme (13), the following results are achieved.

(i) The sliding manifold $\mathbf{S}$ converges to the region $\|\mathbf{S}\| \leq$ $\Delta$ in finite time. Here, $b$ and $b_{1}$ are positive constants satisfying $k_{1}-b>0$ and $b_{1}=f_{\max }^{2} / 4 b$, respectively. $f_{\max }$ is the maximum value of $\left\|\mathbf{E} \mathbf{J}^{-1} \mathbf{d}\right\|$. Consider

$$
\Delta=\min \left\{\left(\frac{b_{1}}{k_{1}-b}\right)^{1 / 2},\left(\frac{b_{1}}{k_{2}}\right)^{1 / 1+\gamma}\right\} .
$$

(ii) The errors $\mathbf{e}_{\widetilde{\mathbf{R}}, i}$ and $\dot{\mathbf{e}}_{\widetilde{\mathbf{R}}, i}$ converge to the regions $\left|\mathbf{e}_{\widetilde{\mathbf{R}}, i}\right| \leq$ $\Delta \mathbf{e}_{\widetilde{\mathbf{R}}}$ and $\left|\dot{\mathbf{e}}_{\widetilde{\mathbf{R}}, i}\right| \leq \Delta \dot{\mathbf{e}}_{\widetilde{\mathbf{R}}}$ in finite time, respectively. Consider

$$
\begin{aligned}
& \Delta \mathbf{e}_{\widetilde{\mathbf{R}}}=\max \left\{\eta, \min \left\{\left(\frac{\Delta}{\alpha}\right)^{1 / 2},\left(\frac{\Delta}{\beta}\right)^{1 /(1+\gamma)}\right\}\right\}, \\
& \Delta \dot{\mathbf{e}}_{\widetilde{\mathbf{R}}}=\Delta+\left(\lambda \ln \frac{1}{1-3 \Delta \mathbf{e}_{\widetilde{\mathbf{R}}}^{2}}+\alpha\right) \Delta \mathbf{e}_{\widetilde{\mathbf{R}}}+\beta \Delta \mathbf{e}_{\widetilde{\mathbf{R}}}^{\gamma} .
\end{aligned}
$$

Proof. We choose the Lyapunov function as $V_{1}=(1 / 2) \mathbf{S}^{T} \mathbf{S}$. Applying (13) and (5)-(7), the derivative of $V_{1}$ can be written as

$$
\begin{aligned}
& \dot{V}_{1}= \mathbf{S}^{T} \dot{\mathbf{S}} \\
&=\mathbf{S}^{T}\left(\dot{\mathbf{E}} \widetilde{\boldsymbol{\omega}}+\mathbf{E} \mathbf{J}^{-1}(\mathbf{F}+\mathbf{u}+\mathbf{d})+\alpha \dot{\mathbf{e}}_{\widetilde{\mathbf{R}}}+\frac{2 \lambda \mathbf{e}_{\widetilde{\mathbf{R}}}^{T} \dot{\mathbf{e}}_{\widetilde{\mathbf{R}}}}{1-\mathbf{e}_{\widetilde{\mathbf{R}}}^{T} \mathbf{e}_{\widetilde{\mathbf{R}}}} \mathbf{e}_{\widetilde{\mathbf{R}}}\right. \\
& \\
&\left.\quad+\lambda\left(\ln \frac{1}{1-\mathbf{e}_{\widetilde{\mathbf{R}}}^{T} \mathbf{e}_{\widetilde{\mathbf{R}}}}\right) \dot{\mathbf{e}}_{\widetilde{\mathbf{R}}}+\beta \dot{\mathbf{f}}\left(\mathbf{e}_{\widetilde{\mathbf{R}}}\right)\right)
\end{aligned}
$$

$$
\begin{aligned}
& \leq-k_{1} \mathbf{S}^{T} \mathbf{S}-k_{2} \mathbf{S}^{T} \operatorname{sig}(\mathbf{S})^{\gamma}+\|\mathbf{S}\|\left\|\mathbf{E J}^{-1} \mathbf{d}\right\| \\
& \leq-\left(k_{1}-b\right) \mathbf{S}^{T} \mathbf{S}-k_{2} \mathbf{S}^{T} \operatorname{sig}(\mathbf{S})^{\gamma}+b_{1} .
\end{aligned}
$$

To deal with $b_{1}, \dot{V}_{1}$ can be rewritten as (18)-(19). We will discuss these situations in Cases 1-2. Consider

$$
\begin{gathered}
\dot{V}_{1} \leq-\left(k_{1}-b-\frac{b_{1}}{\mathbf{S}^{T} \mathbf{S}}\right) \mathbf{S}^{T} \mathbf{S}-k_{2} \mathbf{S}^{T} \operatorname{sig}(\mathbf{S})^{\gamma}, \\
\dot{V}_{1} \leq-\left(k_{1}-b\right) \mathbf{S}^{T} \mathbf{S}-\left(k_{2}-\frac{b_{1}}{\mathbf{S}^{T} \operatorname{sig}(\mathbf{S})^{\gamma}}\right) \mathbf{S}^{T} \operatorname{sig}(\mathbf{S})^{\gamma} .
\end{gathered}
$$

Case 1. Assuming $\eta_{1}=k_{1}-b-b_{1} / \mathbf{S}^{T} \mathbf{S}$ and $\eta_{2}=k_{2}$, (18) can be rewritten as (20). If $\eta_{1}>0$ and $\eta_{2}>0$, the sliding manifold $\mathbf{S}$ will converge to the region $\|\mathbf{S}\| \leq\left(b_{1} /\left(k_{1}-b\right)\right)^{1 / 2}$ in finite time by using Lemma 3. Consider

$$
\dot{V}_{1} \leq-\eta_{1} \mathbf{S}^{T} \mathbf{S}-\eta_{2}\left(\mathbf{S}^{T} \mathbf{S}\right)^{(\gamma+1) / 2} .
$$

Case 2. Assuming $\mu_{1}=k_{1}-b$ and $\mu_{2}=k_{2}-b_{1} / \mathbf{S}^{T} \operatorname{sig}(\mathbf{S})^{\gamma}$, (19) can be rewritten as (21). If $\mu_{1}>0$ and $\mu_{2}>0$, the sliding manifold $\mathbf{S}$ will converge to the region $\|\mathbf{S}\| \leq\left(b_{1} / k_{2}\right)^{1 / 1+\gamma}$ in finite time by using Lemma 3. Consider

$$
\dot{V}_{1} \leq-\mu_{1} \mathbf{S}^{T} \mathbf{S}-\mu_{2}\left(\mathbf{S}^{T} \mathbf{S}\right)^{(\gamma+1) / 2}
$$

Now, (i) has been proved.

The sliding manifold $\mathbf{S}$ converges to the region $\|\mathbf{S}\| \leq \Delta$ in finite time, which means that $\left|S_{i}\right| \leq \Delta, i=1,2,3$. Therefore, the stability analysis of $\mathbf{e}_{\widetilde{\mathbf{R}}, i}$ and $\dot{\mathbf{e}}_{\widetilde{\mathbf{R}}, i}$ is as follows:

Case 1. If $\left|\mathbf{e}_{\widetilde{\mathbf{R}}, i}\right| \leq \eta$, we can get (22) by using (11)-(12). Consider

$$
\begin{aligned}
& S_{i}= \dot{\mathbf{e}}_{\widetilde{\mathbf{R}}, i}+\alpha \mathbf{e}_{\widetilde{\mathbf{R}}, i}+\lambda\left(\ln \frac{1}{1-\mathbf{e}_{\widetilde{\mathbf{R}}}^{T} \mathbf{e}_{\widetilde{\mathbf{R}}}}\right) \mathbf{e}_{\widetilde{\mathbf{R}}, i} \\
&+\beta\left(r_{1} \mathbf{e}_{\widetilde{\mathbf{R}}, i}+r_{2} \operatorname{sign}\left(\mathbf{e}_{\widetilde{\mathbf{R}}, i}\right) \mathbf{e}_{\widetilde{\mathbf{R}}, i}^{2}\right)=\phi_{i}, \quad\left|\phi_{i}\right| \leq \Delta, \\
&\left|\dot{\mathbf{e}}_{\widetilde{\mathbf{R}}, i}\right| \leq \Delta+\left(\lambda \ln \frac{1}{1-3 \eta^{2}}+\alpha\right) \eta+\beta \eta^{\gamma} \leq \Delta \dot{\mathbf{e}}_{\widetilde{\mathbf{R}}} .
\end{aligned}
$$

From (23), we can get that $\dot{\mathbf{e}}_{\widetilde{\mathbf{R}}, i}$ converges to $\left|\dot{\mathbf{e}}_{\widetilde{\mathbf{R}}, i}\right| \leq \Delta \dot{\mathbf{e}}_{\widetilde{\mathbf{R}}}$ in finite time.

Case 2. If $\left|\dot{\mathbf{e}}_{\widetilde{\mathbf{R}}, i}\right|>\eta$, we can get (24) by using (11)-(12). Consider

$$
S_{i}=\dot{\mathbf{e}}_{\widetilde{\mathbf{R}}, i}+\alpha \mathbf{e}_{\widetilde{\mathbf{R}}, i}+\lambda\left(\ln \frac{1}{1-\mathbf{e}_{\widetilde{\mathbf{R}}}^{T} \mathbf{e}_{\widetilde{\mathbf{R}}}}\right) \mathbf{e}_{\widetilde{\mathbf{R}}, i}+\beta \operatorname{sig}\left(\mathbf{e}_{\widetilde{\mathbf{R}}, i}\right)^{\gamma}=\phi_{i} .
$$


We choose the Lyapunov function as $V_{2, i}=(1 / 2) \mathbf{e}_{\widetilde{\mathbf{R}}, i}^{2}$. Applying (24), the derivative of $V_{2, i}$ can be written as follows:

$$
\begin{aligned}
& \dot{V}_{2, i}= \mathbf{e}_{\widetilde{\mathbf{R}}, i} \dot{\mathbf{e}}_{\widetilde{\mathbf{R}}, i} \\
&=-\mathbf{e}_{\widetilde{\mathbf{R}}, i}\left(\alpha \mathbf{e}_{\widetilde{\mathbf{R}}, i}+\lambda\left(\ln \frac{1}{1-\mathbf{e}_{\widetilde{\mathbf{R}}}^{T} \mathbf{e}_{\widetilde{\mathbf{R}}}}\right) \mathbf{e}_{\widetilde{\mathbf{R}}, i}\right. \\
&\left.+\beta \operatorname{sig}\left(\mathbf{e}_{\widetilde{\mathbf{R}}, i}\right)^{\gamma}-\phi_{i}\right) \\
& \leq-\mathbf{e}_{\widetilde{\mathbf{R}}, i}\left(\alpha \mathbf{e}_{\widetilde{\mathbf{R}}, i}+\beta \operatorname{sig}\left(\mathbf{e}_{\widetilde{\mathbf{R}}, i}\right)^{\gamma}-\phi_{i}\right) \\
& \leq-\alpha \mathbf{e}_{\widetilde{\mathbf{R}}, i}^{2}-\beta \mathbf{e}_{\widetilde{\mathbf{R}}, i} \operatorname{sig}\left(\mathbf{e}_{\widetilde{\mathbf{R}}, i}\right)^{\gamma}+\left|\phi_{i}\right| .
\end{aligned}
$$

To deal with $\left|\phi_{i}\right|, \dot{V}_{2, i}$ can be rewritten as follows:

$$
\begin{gathered}
\dot{V}_{2, i} \leq-\left(\alpha-\frac{\left|\phi_{i}\right|}{\mathbf{e}_{\widetilde{\mathbf{R}}, i}^{2}}\right) \mathbf{e}_{\widetilde{\mathbf{R}}, i}^{2}-\beta \mathbf{e}_{\widetilde{\mathbf{R}}, i} \operatorname{sig}\left(\mathbf{e}_{\widetilde{\mathbf{R}}, i}\right)^{\gamma}, \\
\dot{V}_{2, i} \leq-\alpha \mathbf{e}_{\widetilde{\mathbf{R}}, i}^{2}-\left(\beta-\frac{\left|\phi_{i}\right|}{\mathbf{e}_{\widetilde{\mathbf{R}}, i}} \operatorname{sig}\left(\mathbf{e}_{\widetilde{\mathbf{R}}, i}\right)^{\gamma}\right) \mathbf{e}_{\widetilde{\mathbf{R}}, i} \operatorname{sig}\left(\mathbf{e}_{\widetilde{\mathbf{R}}, i}\right)^{\gamma} .
\end{gathered}
$$

If $\alpha-\left|\phi_{i}\right| / \mathbf{e}_{\widetilde{\mathbf{R}}, i}^{2}>0, \mathbf{e}_{\widetilde{\mathbf{R}}, i}$ converges to $\left|\mathbf{e}_{\widetilde{\mathbf{R}}, i}\right| \leq(\Delta / \alpha)^{1 / 2}$ in finite time and if $\beta-\left|\phi_{i}\right| / \mathbf{e}_{\widetilde{\mathbf{R}}, i} \operatorname{sig}\left(\mathbf{e}_{\widetilde{\mathbf{R}}, i}\right)^{\gamma}>0, \mathbf{e}_{\widetilde{\mathbf{R}}, i}$ converges to $\left|\mathbf{e}_{\widetilde{\mathbf{R}}, i}\right| \leq(\Delta / \beta)^{1 /(1+\gamma)}$ in finite time. Consider

$$
\begin{aligned}
\left|\dot{\mathbf{e}}_{\widetilde{\mathbf{R}}, i}\right| \leq & \left|\phi_{i}\right|+\left(\lambda \ln \left(\frac{1}{1-\mathbf{e}_{\widetilde{\mathbf{R}}}^{T} \mathbf{e}_{\widetilde{\mathbf{R}}}}\right)+\alpha\right)\left|\mathbf{e}_{\widetilde{\mathbf{R}}, i}\right| \\
& +\beta\left|\mathbf{e}_{\widetilde{\mathbf{R}}, i}\right|^{\gamma}=\Delta \dot{\mathbf{e}}_{\widetilde{\mathbf{R}}} .
\end{aligned}
$$

Furthermore, from (27) we can get that $\dot{\mathbf{e}}_{\widetilde{\mathbf{R}}, i}$ converges to $\left|\dot{\mathbf{e}}_{\widetilde{\mathbf{R}}, i}\right| \leq \Delta \dot{\mathbf{e}}_{\widetilde{\mathbf{R}}}$ in finite time.

Now, (ii) has been proved.

Remark 6. From (14)-(15), it is concluded that the controller parameters $k_{1}$ and $k_{2}$ determine the accuracy of $S_{i}$ and the controller parameters $\alpha$ and $\beta$ determine the final accuracy of $\mathbf{e}_{\widetilde{\mathbf{R}}, i}$. In order to get small $S_{i}$ and $\mathbf{e}_{\widetilde{\mathbf{R}}, i}$, we can select large $k_{1}$, $k_{2}, \alpha$, and $\beta$.

In Theorem 5 , it is assumed that $d_{\max }$ is given. However, in applications, $d_{\max }$ is hard to know. In order to deal with a time-varying unknown bounded disturbance, we design the discontinuous controller (28)-(29), where $\widehat{d}_{\max }$ is the estimation value of $d_{\max }$ and $\widetilde{d}_{\max }=d_{\max }-\widehat{d}_{\max }$. Consider

$$
\begin{gathered}
\mathbf{u}=-\mathbf{J} \mathbf{E}^{-1}\left(\dot{\mathbf{E}} \widetilde{\boldsymbol{\omega}}+\alpha \dot{\mathbf{e}}_{\widetilde{\mathbf{R}}}+\frac{2 \lambda \mathbf{e}_{\widetilde{\mathbf{R}}}^{T} \dot{\mathbf{e}}_{\widetilde{\mathbf{R}}}}{1-\mathbf{e}_{\widetilde{\mathbf{R}}}^{T} \mathbf{e}_{\widetilde{\mathbf{R}}}} \mathbf{e}_{\widetilde{\mathbf{R}}}+\lambda \ln \frac{1}{1-\mathbf{e}_{\widetilde{\mathbf{R}}}^{T} \mathbf{e}_{\widetilde{\mathbf{R}}}} \dot{\mathbf{e}}_{\widetilde{\mathbf{R}}}\right. \\
\left.+\beta \dot{\mathbf{f}}\left(\mathbf{e}_{\widetilde{\mathbf{R}}}\right)+k_{1} \mathbf{S}+k_{2} \operatorname{sign}(\mathbf{S})\right) \\
-\widehat{d}_{\max } \operatorname{sign}\left(\mathbf{J}^{-T} \mathbf{E}^{T} \mathbf{S}\right)-\mathbf{F}, \\
\dot{\vec{d}}_{\max }=\left\|\mathbf{S}^{T} \mathbf{E J}^{-1}\right\| .
\end{gathered}
$$

Theorem 7. Consider a spacecraft described by (5)-(6) under Assumption 4, where $d_{\max }$ is an unknown positive constant. By applying the proposed control scheme (28)-(29), $\mathbf{S}$ and $\widetilde{d}_{\max }$ are all bounded.

Proof. We choose the Lyapunov function as $V_{3}=(1 / 2) \mathbf{S}^{T} \mathbf{S}+$ $(1 / 2) \tilde{d}_{\max }^{2}$. Applying (28)-(29) and (5)-(7), the derivative of $V_{3}$ can be written as follows:

$$
\begin{aligned}
\dot{V}_{3}= & \mathbf{S}^{\mathrm{T}} \dot{\mathbf{S}}+\widetilde{d}_{\max } \dot{\vec{d}}_{\max } \\
= & \mathbf{S}^{T}\left(\dot{\mathbf{E}} \widetilde{\boldsymbol{\omega}}+\mathbf{E J}^{-1}(\mathbf{F}+\mathbf{u}+\mathbf{d})+\frac{2 \lambda \mathbf{e}_{\widetilde{\mathbf{R}}}^{T} \dot{\mathbf{e}}_{\widetilde{\mathbf{R}}}}{1-\mathbf{e}_{\widetilde{\mathbf{R}}}^{T} \mathbf{e}_{\widetilde{\mathbf{R}}}} \mathbf{e}_{\widetilde{\mathbf{R}}}\right. \\
& \left.\quad+\alpha \dot{\mathbf{e}}_{\widetilde{\mathbf{R}}}+\lambda \ln \frac{1}{1-\mathbf{e}_{\widetilde{\mathbf{R}}}^{T} \mathbf{e}_{\widetilde{\mathbf{R}}}} \dot{\mathbf{e}}_{\widetilde{\mathbf{R}}}+\beta \dot{\mathbf{f}}\left(\mathbf{e}_{\widetilde{\mathbf{R}}}\right)\right)-\widetilde{d}_{\max } \dot{\vec{d}}_{\max } \\
\leq & -k_{1} \mathbf{S}^{T} \mathbf{S}-k_{2} \mathbf{S}^{T} \operatorname{sign}(\mathbf{S})+\left\|\mathbf{S}^{T} \mathbf{E} \mathbf{J}^{-1}\right\| \widetilde{d}_{\max }-\widetilde{d}_{\max } \dot{\vec{d}}_{\max } \\
\leq & 0 .
\end{aligned}
$$

It can be seen that $\dot{V}_{3} \leq 0$. Thus, it can be concluded that variables $\mathbf{S}$ and $\widetilde{d}_{\max }$ are all bounded.

Theorem 8. Consider a spacecraft described by (5)-(6) under Assumption 4, where $d_{\max }$ is an unknown positive constant. By applying the proposed control scheme (28)-(29), the following results are achieved.

(i) The sliding manifold $\mathbf{S}$ converges to $\mathbf{S}=0$ in finite time.

(ii) The errors $\mathbf{e}_{\widetilde{\mathbf{R}}, i}$ and $\dot{\mathbf{e}}_{\widetilde{\mathbf{R}}, i}$ converge to the regions $\left|\mathbf{e}_{\widetilde{\mathbf{R}}, i}\right| \leq$ $\Delta_{1} \mathbf{e}_{\widetilde{\mathbf{R}}}$ and $\left|\dot{\mathbf{e}}_{\widetilde{\mathbf{R}}, i}\right| \leq \Delta_{1} \dot{\mathbf{e}}_{\widetilde{\mathbf{R}}}$ in finite time, respectively. Consider

$$
\Delta_{1} \mathbf{e}_{\widetilde{\mathbf{R}}}=\eta
$$

$$
\Delta_{1} \dot{\mathbf{e}}_{\widetilde{\mathbf{R}}}=\left(\lambda \ln \frac{1}{1-3 \Delta_{1} \mathbf{e}_{\widetilde{\mathbf{R}}}^{2}}+\alpha\right) \Delta_{1} \mathbf{e}_{\widetilde{\mathbf{R}}}+\beta \Delta_{1} \mathbf{e}_{\widetilde{\mathbf{R}}}^{\gamma} .
$$


Proof. We choose the Lyapunov function as $V_{4}=(1 / 2) \mathbf{S}^{T} \mathbf{S}$. Applying (28)-(29) and (5)-(7), the derivative of $V_{4}$ can be written as

$$
\begin{aligned}
\dot{V}_{4}= & \mathbf{S}^{T} \dot{\mathbf{S}} \\
= & \mathbf{S}^{T}\left(\dot{\mathbf{E}} \widetilde{\boldsymbol{\omega}}+\mathbf{E J}^{-1}(\mathbf{F}+\mathbf{d})+\frac{2 \lambda \mathbf{e}_{\widetilde{\mathbf{R}}}^{T} \dot{\mathbf{e}}_{\widetilde{\mathbf{R}}}}{1-\mathbf{e}_{\widetilde{\mathbf{R}}}^{T} \mathbf{e}_{\widetilde{\mathbf{R}}}} \mathbf{e}_{\widetilde{\mathbf{R}}}\right. \\
& \left.\quad+\alpha \dot{\mathbf{e}}_{\widetilde{\mathbf{R}}}+\lambda \ln \frac{1}{1-\mathbf{e}_{\widetilde{\mathbf{R}}}^{T} \mathbf{e}_{\widetilde{\mathbf{R}}}} \dot{\mathbf{e}}_{\widetilde{\mathbf{R}}}+\beta \dot{\mathbf{f}}\left(\mathbf{e}_{\widetilde{\mathbf{R}}}\right)\right)+\mathbf{S}^{T} \mathbf{E J}^{-1} \mathbf{u} \\
\leq & -k_{1} \mathbf{S}^{T} \mathbf{S}-\left(k_{2}-\widetilde{d}_{\max }\right)\|\mathbf{S}\| .
\end{aligned}
$$

If $k_{2}-\tilde{d}_{\max }>0$, then, by using Lemma 3 , we can get that the sliding manifold $\mathbf{S}$ will converge to $\mathbf{S}=0$ in finite time.

Therefore, the stability analysis of $\mathbf{e}_{\widetilde{\mathbf{R}}, i}$ and $\dot{\mathbf{e}}_{\widetilde{\mathbf{R}}, i}$ is as follows.

Case 1. If $\left|\mathbf{e}_{\widetilde{\mathrm{R}}, i}\right| \leq \eta$, we can get (34) by using (11)-(12). Consider

$$
\begin{aligned}
S_{i}= & \dot{\mathbf{e}}_{\widetilde{\mathbf{R}}, i}+\alpha \mathbf{e}_{\widetilde{\mathbf{R}}, i}+\lambda\left(\ln \frac{1}{1-\mathbf{e}_{\widetilde{\mathbf{R}}}^{T} \mathbf{e}_{\widetilde{\mathbf{R}}}}\right) \mathbf{e}_{\widetilde{\mathbf{R}}, i} \\
& +\beta\left(r_{1} \mathbf{e}_{\widetilde{\mathbf{R}}, i}+r_{2} \operatorname{sign}\left(\mathbf{e}_{\widetilde{\mathbf{R}}, i}\right) \mathbf{e}_{\widetilde{\mathbf{R}}, i}^{2}\right)=0, \\
\left|\dot{\mathbf{e}}_{\widetilde{\mathbf{R}}, i}\right| \leq & \left(\lambda \ln \frac{1}{1-3 \eta^{2}}+\alpha\right) \eta+\beta \eta^{\gamma} \leq \Delta_{1} \dot{\mathbf{e}}_{\widetilde{\mathbf{R}}} .
\end{aligned}
$$

From (35), we can get that $\dot{\mathbf{e}}_{\widetilde{\mathbf{R}}, i}$ converges to $\left|\dot{\mathbf{e}}_{\widetilde{\mathbf{R}}, i}\right| \leq \Delta_{1} \dot{\mathbf{e}}_{\widetilde{\mathbf{R}}}$ in finite time.

Case 2. If $\left|\dot{\mathbf{e}}_{\widetilde{\mathbf{R}}, i}\right|>\eta$, we can get (36) by using (11)-(12). Consider

$$
S_{i}=\dot{\mathbf{e}}_{\widetilde{\mathbf{R}}, i}+\alpha \mathbf{e}_{\widetilde{\mathbf{R}}, i}+\lambda\left(\ln \frac{1}{1-\mathbf{e}_{\widetilde{\mathbf{R}}}^{T} \mathbf{e}_{\widetilde{\mathbf{R}}}}\right) \mathbf{e}_{\widetilde{\mathbf{R}}, i}+\beta \operatorname{sig}\left(\mathbf{e}_{\widetilde{\mathbf{R}}, i}\right)^{\gamma}=0 .
$$

We choose the Lyapunov function as $V_{5, i}=(1 / 2) \mathbf{e}_{\widetilde{\mathbf{R}}, i}^{2}$. Applying (36), the derivative of $V_{5, i}$ can be written as follows:

$$
\begin{aligned}
\dot{V}_{5, i} & =\mathbf{e}_{\widetilde{\mathbf{R}}, i} \dot{\mathbf{e}}_{\widetilde{\mathbf{R}}, i} \\
& =-\mathbf{e}_{\widetilde{\mathbf{R}}, i}\left(\alpha \mathbf{e}_{\widetilde{\mathbf{R}}, i}+\lambda\left(\ln \frac{1}{1-\mathbf{e}_{\widetilde{\mathbf{R}}}^{T} \mathbf{e}_{\widetilde{\mathbf{R}}}}\right) \mathbf{e}_{\widetilde{\mathbf{R}}, i}+\beta \operatorname{sig}\left(\mathbf{e}_{\widetilde{\mathbf{R}}, i}\right)^{\gamma}\right) \\
& \leq-\mathbf{e}_{\widetilde{\mathbf{R}}, i}\left(\alpha \mathbf{e}_{\widetilde{\mathbf{R}}, i}+\beta \operatorname{sig}\left(\mathbf{e}_{\widetilde{\mathbf{R}}, i}\right)_{i}^{\gamma}\right) \\
& \leq-\alpha \mathbf{e}_{\widetilde{\mathbf{R}}, i}^{2}-\beta \mathbf{e}_{\widetilde{\mathbf{R}}, i} \operatorname{sig}\left(\mathbf{e}_{\widetilde{\mathbf{R}}, i}\right)^{\gamma} .
\end{aligned}
$$

It can be seen that $\mathbf{e}_{\widetilde{\mathbf{R}}, i}$ converges to $\left|\mathbf{e}_{\widetilde{\mathbf{R}}, i}\right| \leq \eta$ in finite time. Consider

$$
\left|\dot{\mathbf{e}}_{\widetilde{\mathbf{R}}, i}\right| \leq\left(\lambda \ln \left(\frac{1}{1-\mathbf{e}_{\widetilde{\mathbf{R}}}^{T} \mathbf{e}_{\widetilde{\mathbf{R}}}}\right)+\alpha\right)\left|\mathbf{e}_{\widetilde{\mathbf{R}}, i}\right|+\beta\left|\mathbf{e}_{\widetilde{\mathbf{R}}, i}\right|^{\gamma}=\Delta_{1} \dot{\mathbf{e}}_{\widetilde{\mathbf{R}}} .
$$

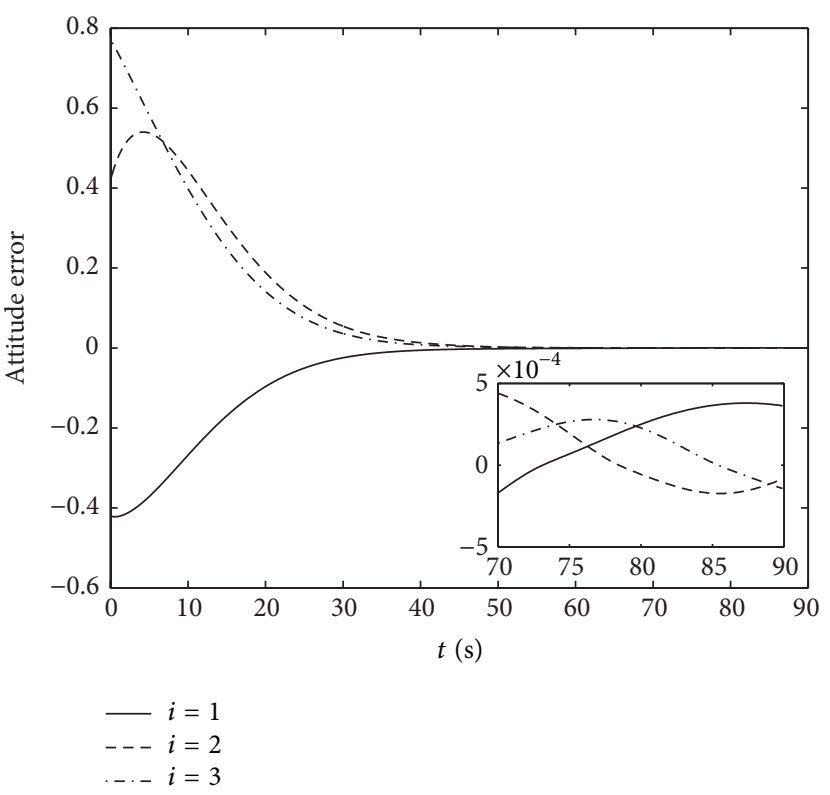

Figure 1: The curves of $\mathbf{e}_{\widetilde{\mathbf{R}}}$ under the first controller.

Furthermore, from (38), we can get that $\dot{\mathbf{e}}_{\widetilde{\mathbf{R}}, i}$ converges to $\left|\dot{\mathbf{e}}_{\widetilde{\mathbf{R}}, i}\right| \leq \Delta_{1} \dot{\mathbf{e}}_{\widetilde{\mathbf{R}}}$ in finite time.

Now, (ii) has been proved.

Now, Theorem 8 has been proved.

Remark 9. In Theorems 7 and $8, \widetilde{d}_{\max }$ does not converge to the region near zero in finite time. It just guarantees that $\widetilde{d}_{\max }$ is bounded.

Remark 10. Owing to the novel modified nonsingular fast terminal sliding manifold, we can easily ensure that $\operatorname{tr}(\widetilde{\mathbf{R}}) \neq$ -1 from the proofs. Compared with $[10,18]$, we do not need to consider the region of attraction in the controllers. Because the system initial value can not be $\operatorname{tr}(\widetilde{\mathbf{R}}) \neq-1$, the two controllers are almost globally finite-time stabilization.

\section{Simulations}

In this section, the simulation is given to illustrate the theoretical results. To validate the effectiveness of the proposed two controllers, numerical simulations are given in the following scenario. The spacecraft tracks a time-varying reference signal.

The model parameters and initial value for the spacecraft are defined as follows:

$$
\begin{aligned}
\mathbf{J} & =\left[\begin{array}{ccc}
22.7 & 0.2 & -0.5 \\
0.2 & 23.3 & 0.3 \\
-0.5 & 0.3 & 24.5
\end{array}\right] \mathrm{kg} \cdot \mathrm{m}^{2}, \\
\mathbf{R}(0) & =\left[\begin{array}{ccc}
-0.5414 & -0.7072 & -0.4546 \\
-0.0009 & -0.5403 & 0.8415 \\
-0.8407 & 0.4560 & 0.2919
\end{array}\right], \\
& \boldsymbol{\omega}(0)=[0.1,0.1,0.1] \mathrm{rad} / \mathrm{s} .
\end{aligned}
$$




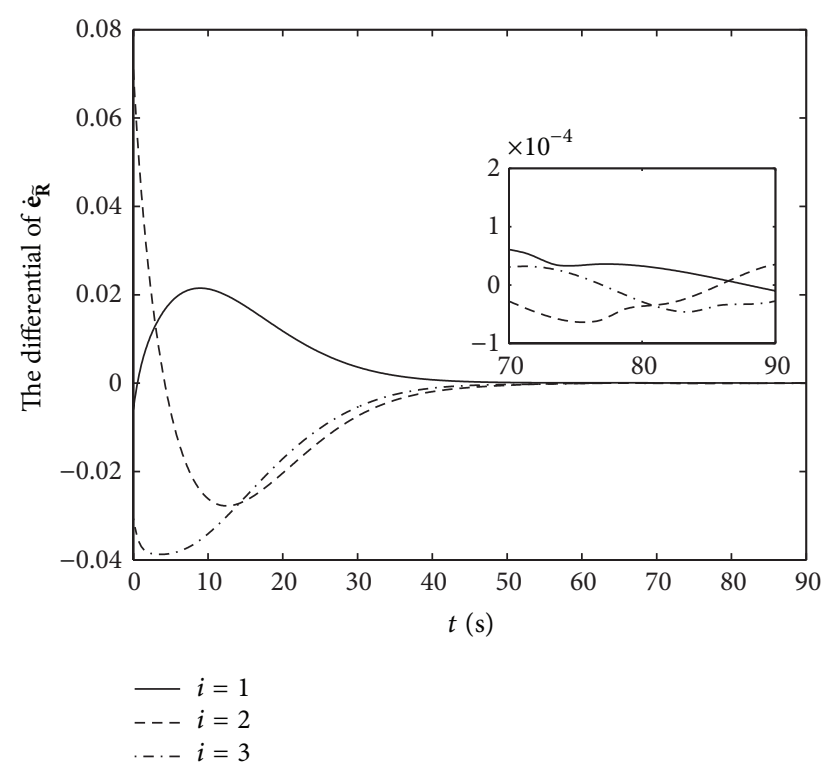

FIGURE 2: The curves of $\dot{\mathbf{e}}_{\widetilde{\mathbf{R}}}$ under the first controller.

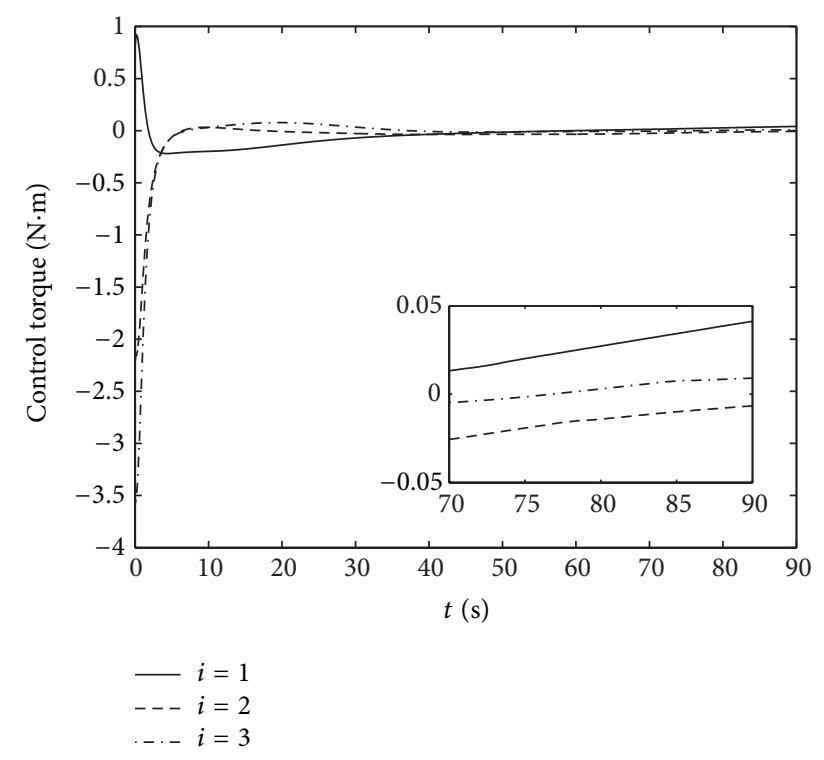

FIGURE 3: The control torques of the system under the first controller.

The initial value and reference angular velocity for the spacecraft are as follows:

$$
\begin{gathered}
\mathbf{R}_{d}(0)=\mathbf{I} \\
\boldsymbol{\omega}_{d}=\left[0.1 \sin \left(\frac{t}{40}\right),-0.1 \cos \left(\frac{t}{50}\right),-0.1 \sin \left(\frac{t}{60}\right)\right]^{T} \mathrm{rad} / \mathrm{s} .
\end{gathered}
$$

For the dynamic model described by (5) and (6), it should be noted that system exists external disturbance. The disturbance torque $\mathbf{d}$ in (6) is defined as follows:

$$
\mathbf{d}=2 \times 10^{-3}[\sin (0.1 t), \cos (0.2 t), \sin (0.2 t)]^{T} \mathrm{~N} \cdot \mathrm{m} .
$$

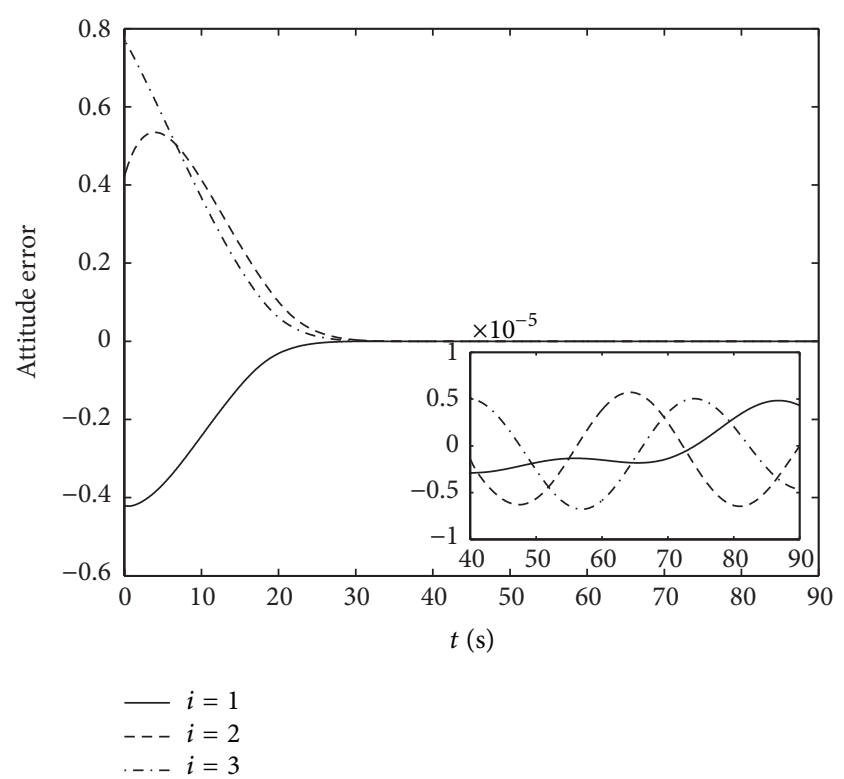

FIGURE 4: The curves of $\mathbf{e}_{\widetilde{\mathrm{R}}}$ under the second controller.

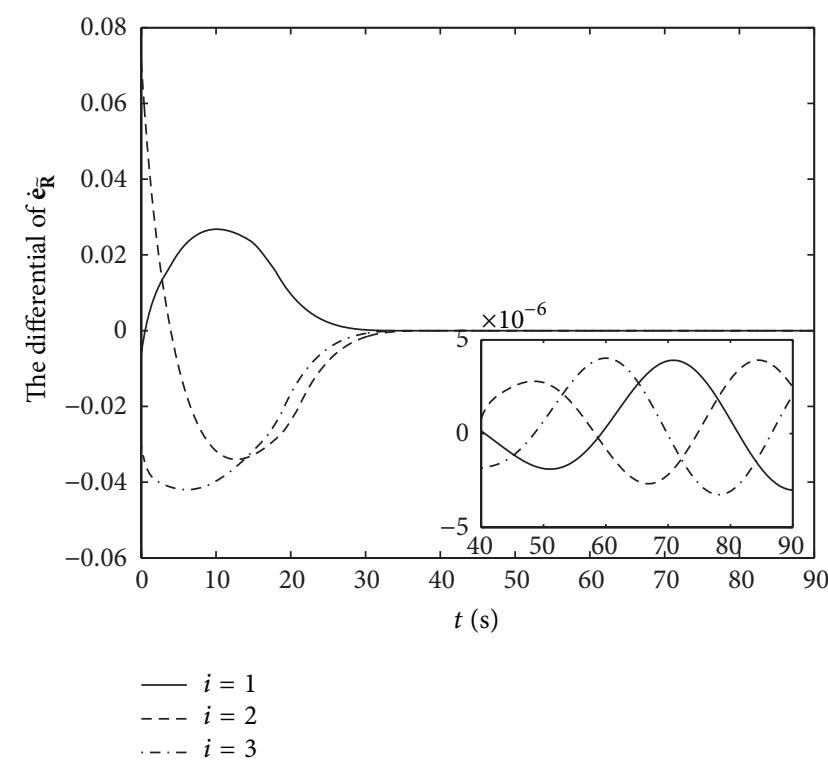

FIgURE 5: The curves of $\dot{\mathbf{e}}_{\widetilde{\mathbf{R}}}$ under the second controller.

We select parameters of the first controller as $\alpha=0.01$, $\beta=0.15, k_{1}=0.1, k_{2}=0.001$, and $\gamma=0.8$. Simulation results of the spacecraft system under the controller (13) are shown in Figures 1-3, from which it can be seen that the maneuver can be completed in less than 70 seconds. Figure 1 gives the attitude curves of the spacecraft. As $\operatorname{tr}(\widetilde{\mathbf{R}}(0)) \neq-1$, the controller (13) is effective. Figure 2 gives the cures of $\dot{\mathbf{e}}_{\widetilde{\mathbf{R}}}$. Figure 3 plots the curves of control torque of the system, from which it can be seen that the controller is continuous without chattering. It is easy to find that the controller (13) can obtain better performance when the attitude tracking is performed.

To validate the second controller, numerical simulations are given as follows. The parameters of the second controller 


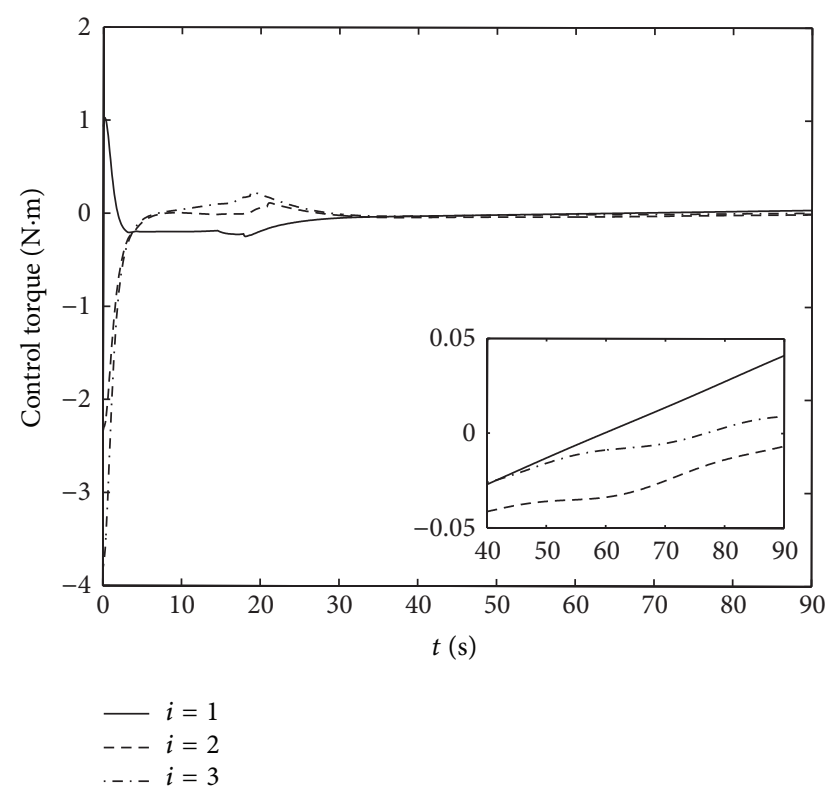

Figure 6: The control torques of the system under the second controller.

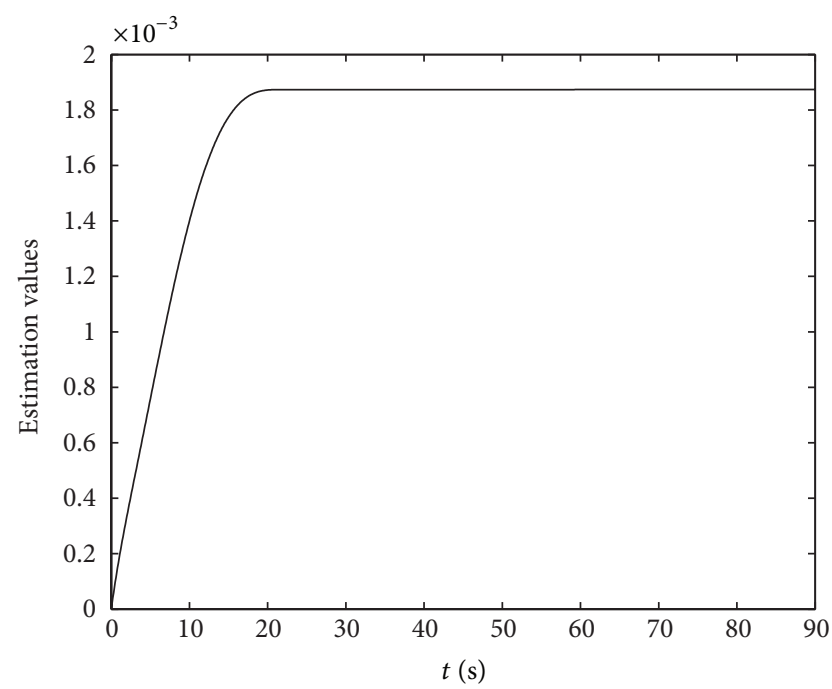

FIGURE 7: The estimated value of the disturbance torque under the second controller.

are selected the same as the first one. In order to avoid chattering, we use saturation function to take the place of sign function. Simulation results of the spacecraft system under the controller (28) are shown in Figures 4, 5, 6, and 7. We can see that the attitude maneuver of the spacecraft can be completed in less than 40 seconds. Compared with Figures 3 and 6 , it can be seen that the control torques of the both controllers are similar. Compared with Figures 1 and 4, the second controller has more excellent performance than the first controller.

In order to get similar performance as the second controller, the parameters of the first controller are chosen as $\alpha=0.01, \beta=0.15, k_{1}=0.1, k_{2}=1$, and $\gamma=0.8$. Figure 8 gives the attitude curves of the

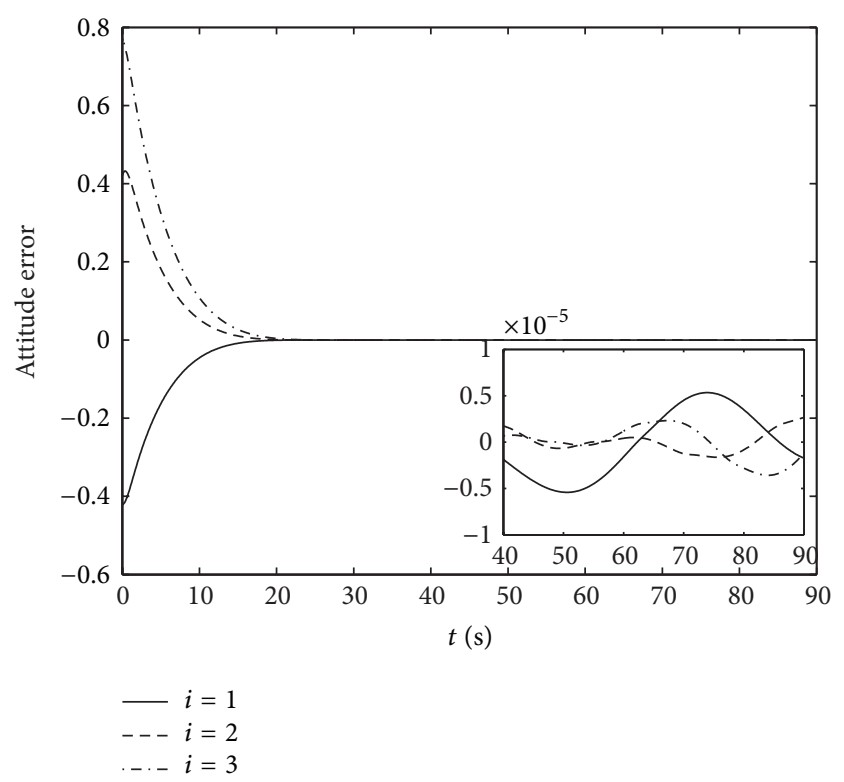

FIGURE 8: The curves of $\mathbf{e}_{\widetilde{\mathbf{R}}}$ under the first controller with $k_{2}=1$.

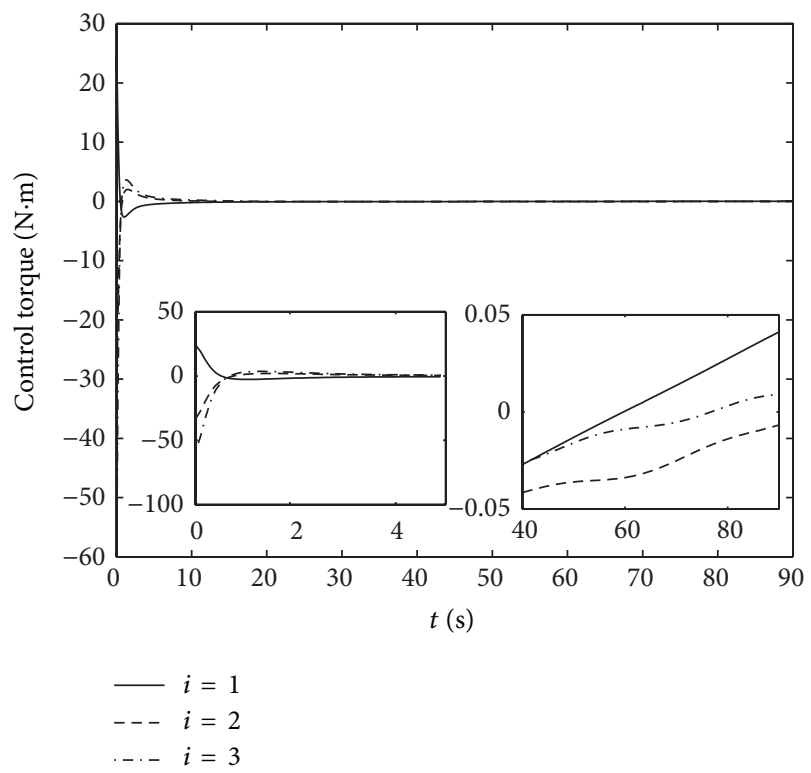

FIGURE 9: The control torques of the system under the first controller with $k_{2}=1$.

spacecraft. Figure 9 gives the curves of control torque of the system. Compared with Figures 6 and 9, the first controller needs larger control torques. The results of simulation show that both controllers can obtain better performance when the attitude tracking is performed and the second controller has more excellent performance than the first controller.

To further research, the first controller is compared with the controller (21)-(23) in [18]. We chose the parameters of the first controller as $\alpha=0.01, \beta=0.23, k_{1}=0.35$, $k_{2}=0.002$, and $\gamma=0.8$ to get similar performance as the controller in [18]. Figures 10 and 11 give curves of the 


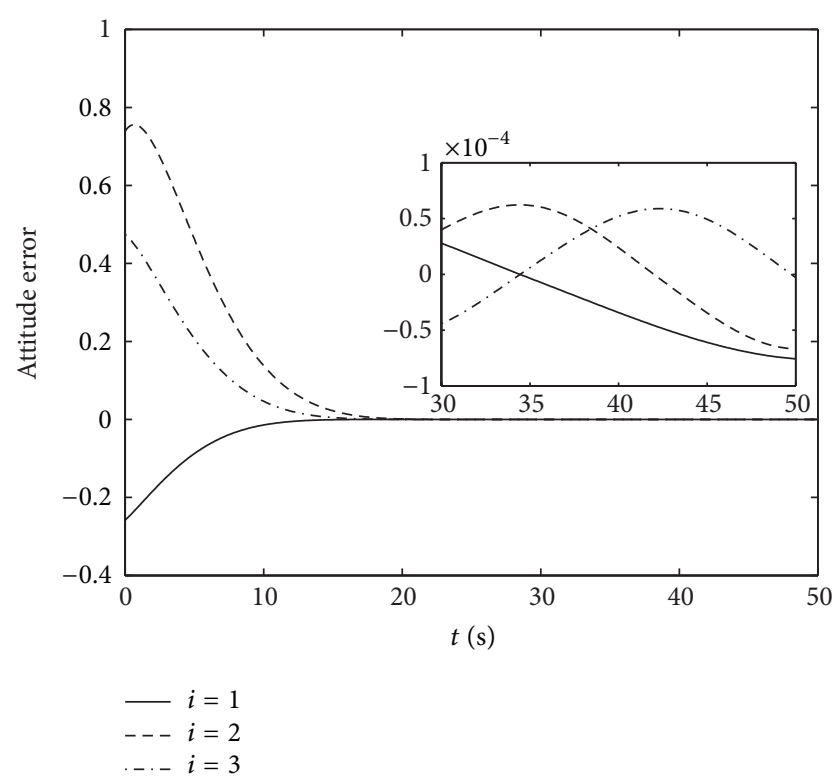

FIgURE 10: The curves of $\mathbf{e}_{\widetilde{\mathrm{R}}}$ under the first controller with $k_{2}=$ 0.002 .

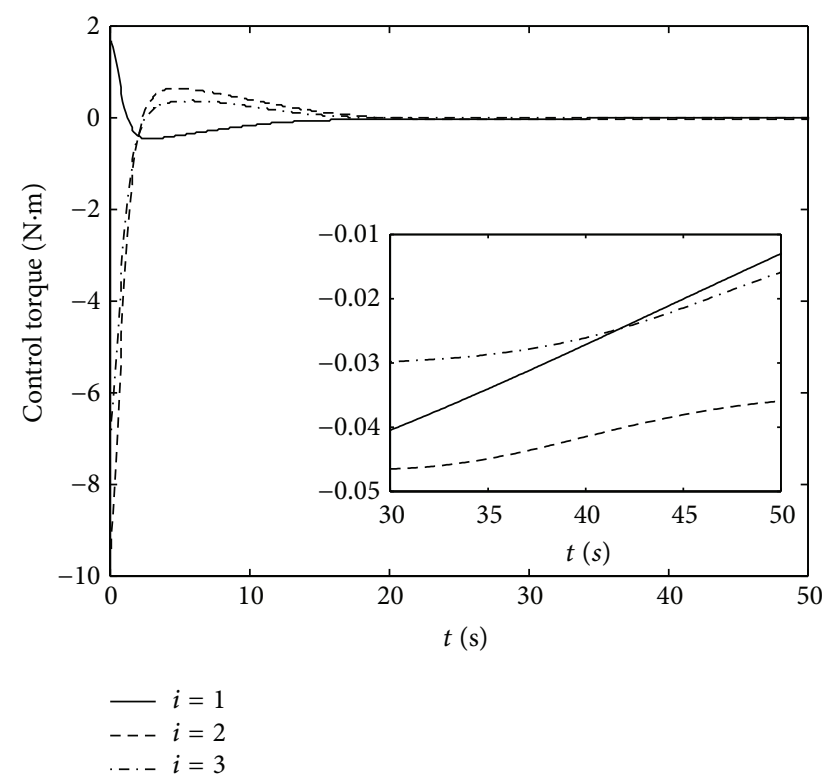

FIgURE 11: The control torques of the system under the first controller with $k_{2}=0.002$.

attitude and control torque of the spacecraft. Figures 12 and 13 give the corresponding curves of the controller in [18]. Compared with Figures 11 and 13, the first controller needs smaller control torques. We can see that both controllers can obtain better performance when the attitude tracking is performed and the first controller outperforms the controller in [18] clearly.

\section{Conclusions}

This work develops two robust finite-time controllers without unwinding for the spacecraft. The first proposed robust

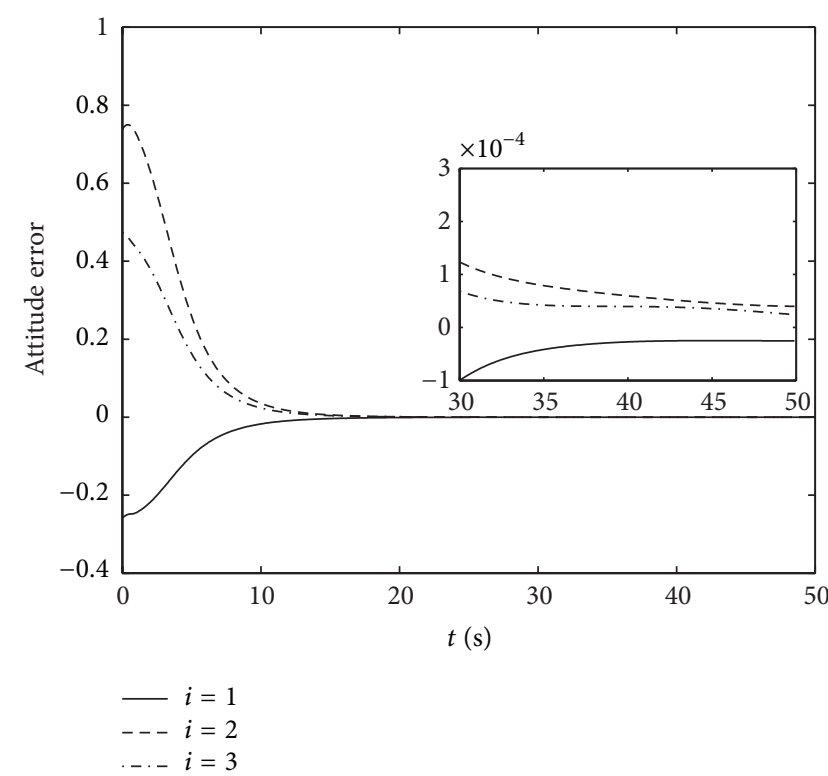

FIgURE 12: The curves of $\mathbf{e}_{\widetilde{\mathbf{R}}}$ under the controller in [18].

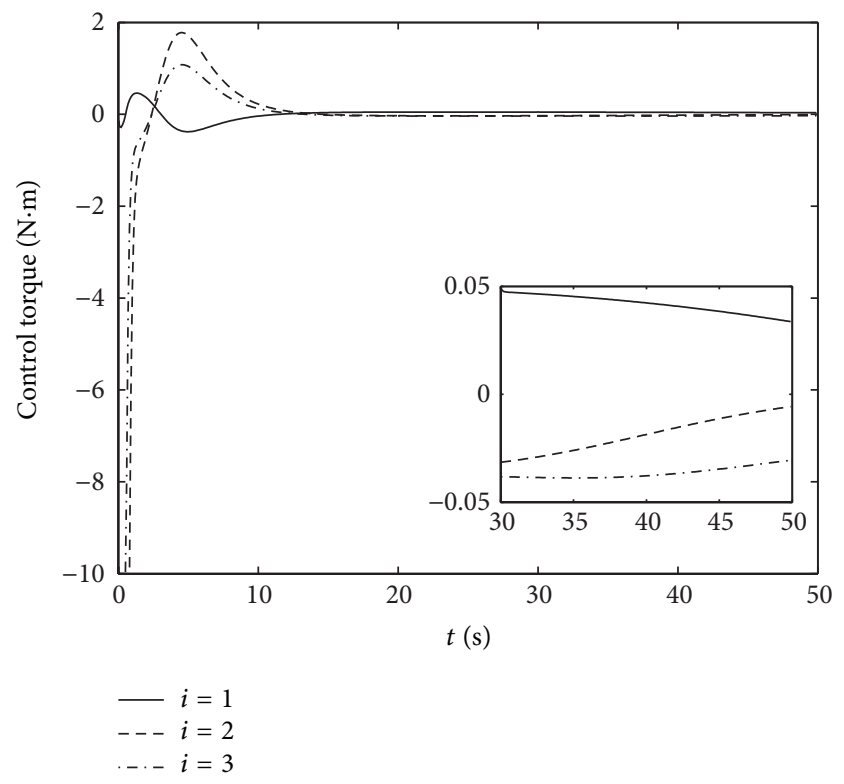

FIGURE 13: The control torques of the system under the controller in [18].

controller is continuous to avoid the chattering. With the use of adaptive control, the second proposed robust controller does not need the bounds of external disturbances. For both controllers, we can get that the overall closedloop system is finite-time stability by Lyapunov's theorems. Simulations have shown that the controllers can make the spacecraft follow a time-varying reference attitude signal without unwinding in finite time.

\section{Conflict of Interests}

The authors declare that there is no conflict of interests regarding the publication of this paper. 


\section{Acknowledgments}

The authors would like to acknowledge the financial support provided by the National Natural Science Foundation of China (Grant no. 61174037), the State Key Program of National Natural Science of China (no. NSFC-61333003), the Innovative Team Program of the National Natural Science Foundation of China (Grant no. 61321062), CAST20120602.

\section{References}

[1] J. T. Wen and K. Kreutz-Delgado, "The attitude control problem," IEEE Transactions on Automatic Control, vol. 36, no. 10, pp. 1148-1162, 1991.

[2] R. Schlanbusch, A. Loria, and P. J. Nicklasson, "On the stability and stabilization of quaternion equilibria of rigid bodies," Automatica, vol. 48, no. 12, pp. 3135-3141, 2012.

[3] J. Wang, H. Liang, and Z. Sun, "Dual-quaternion-based finitetime control for spacecraft tracking in six degrees of freedom," Proceedings of the Institution of Mechanical Engineers, Part G: Journal of Aerospace Engineering, vol. 227, no. 3, pp. 528-545, 2013.

[4] B.-Q. Zhang, S.-M. Song, and X.-L. Chen, "Decentralized robust coordinated control for formation flying spacecraft with coupled attitude and translational dynamics," Proceedings of the Institution of Mechanical Engineers, Part G: Journal of Aerospace Engineering, vol. 227, no. 5, pp. 798-815, 2013.

[5] S. Song, B. Zhang, X. Wei, and X. Chen, "Asymptotical stability analysis of "PD+" controller for spacecraft attitude tracking system," in Proceedings of the 8th World Congress on Intelligent Control and Automation (WCICA '10), pp. 3908-3913, Jinan, China, July 2010.

[6] N. A. Chaturvedi, A. K. Sanyal, and N. H. McClamroch, "Rigidbody attitude control: using rotation matrices for continuous, singularity-free control laws," IEEE Control Systems Magazine, vol. 31, no. 3, pp. 30-51, 2011.

[7] A. Weiss, X. Yang, I. Kolmanovsky, and D. S. Bernstein, "Inertiafree spacecraft attitude control with reaction-wheel actuation," in Proceedings of the AIAA Guidance, Navigation, and Control Conference, Toronto, Canada, August 2010.

[8] A. Sanyal, A. Fosbury, N. Chaturvedi, and D. S. Bernstein, "Inertia-free spacecraft attitude tracking with disturbance rejection and almost global stabilization," Journal of Guidance, Control, and Dynamics, vol. 32, no. 4, pp. 1167-1178, 2009.

[9] N. A. Chaturvedi, N. H. McClamroch, and D. S. Bernstein, "Asymptotic smooth stabilization of the inverted 3-D pendulum," IEEE Transactions on Automatic Control, vol. 54, no. 6, pp. 1204-1215, 2009.

[10] T. Lee, "Exponential stability of an attitude tracking control system on $\mathrm{SO}_{3}$ for large-angle rotational maneuvers," Systems \& Control Letters, vol. 61, no. 1, pp. 231-237, 2012.

[11] Y. Feng, X. Yu, and Z. H. Man, "Non-singular terminal sliding mode control of rigid manipulators," Automatica, vol. 38, no. 12, pp. 2159-2167, 2002.

[12] X. Yu and M. Zhihong, "Fast terminal sliding-mode control design for nonlinear dynamical systems," IEEE Transactions on Circuits and Systems. I. Fundamental Theory and Applications, vol. 49, no. 2, pp. 261-264, 2002.

[13] S. Yu, X. Yu, B. Shirinzadeh, and Z. Man, "Continuous finitetime control for robotic manipulators with terminal sliding mode," Automatica, vol. 41, no. 11, pp. 1957-1964, 2005.
[14] S. Wu, G. Radice, Y. Gao, and Z. Sun, "Quaternion-based finite time control for spacecraft attitude tracking," Acta Astronautica, vol. 69, no. 1-2, pp. 48-58, 2011.

[15] K. Lu and Y. Xia, "Finite-time fault-tolerant control for rigid spacecraft with actuator saturations," IET Control Theory \& Applications, vol. 7, no. 11, pp. 1529-1539, 2013.

[16] A. M. Zou and K. D. Kumar, "Distributed attitude coordination control for spacecraft formation flying," IEEE Transactions on Aerospace and Electronic Systems, vol. 48, no. 2, pp. 1329-1346, 2012.

[17] C. Pukdeboon, "Finite-time second-order sliding mode controllers for spacecraft attitude tracking," Mathematical Problems in Engineering, vol. 2013, Article ID 930269, 12 pages, 2013.

[18] Y. Guo and S. Song, "Adaptive finite-time backstepping control for attitude tracking of spacecraft based on rotation matrix," Chinese Journal of Aeronautics, vol. 27, no. 2, pp. 375-382, 2014. 


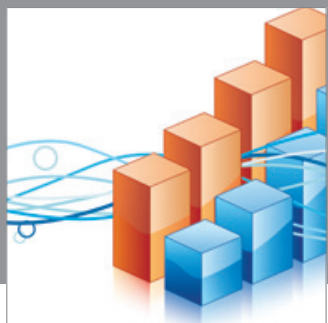

Advances in

Operations Research

mansans

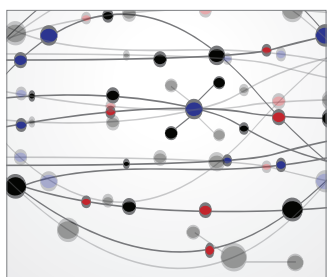

The Scientific World Journal
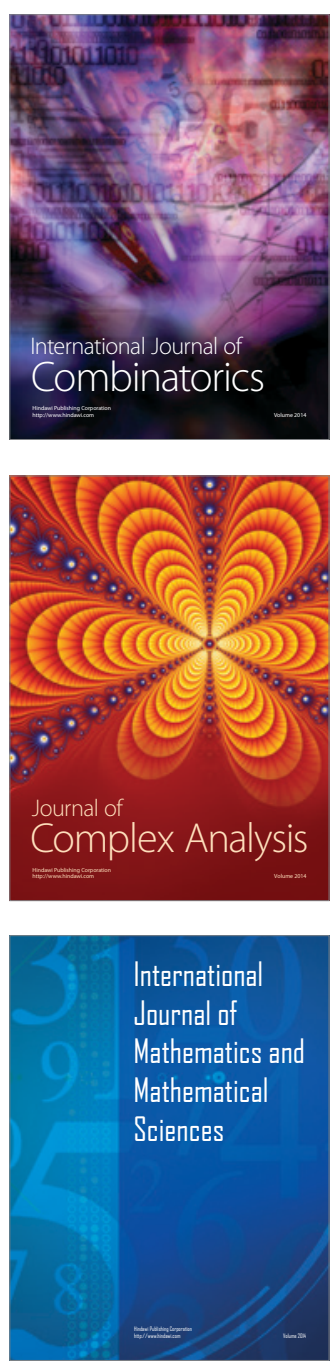
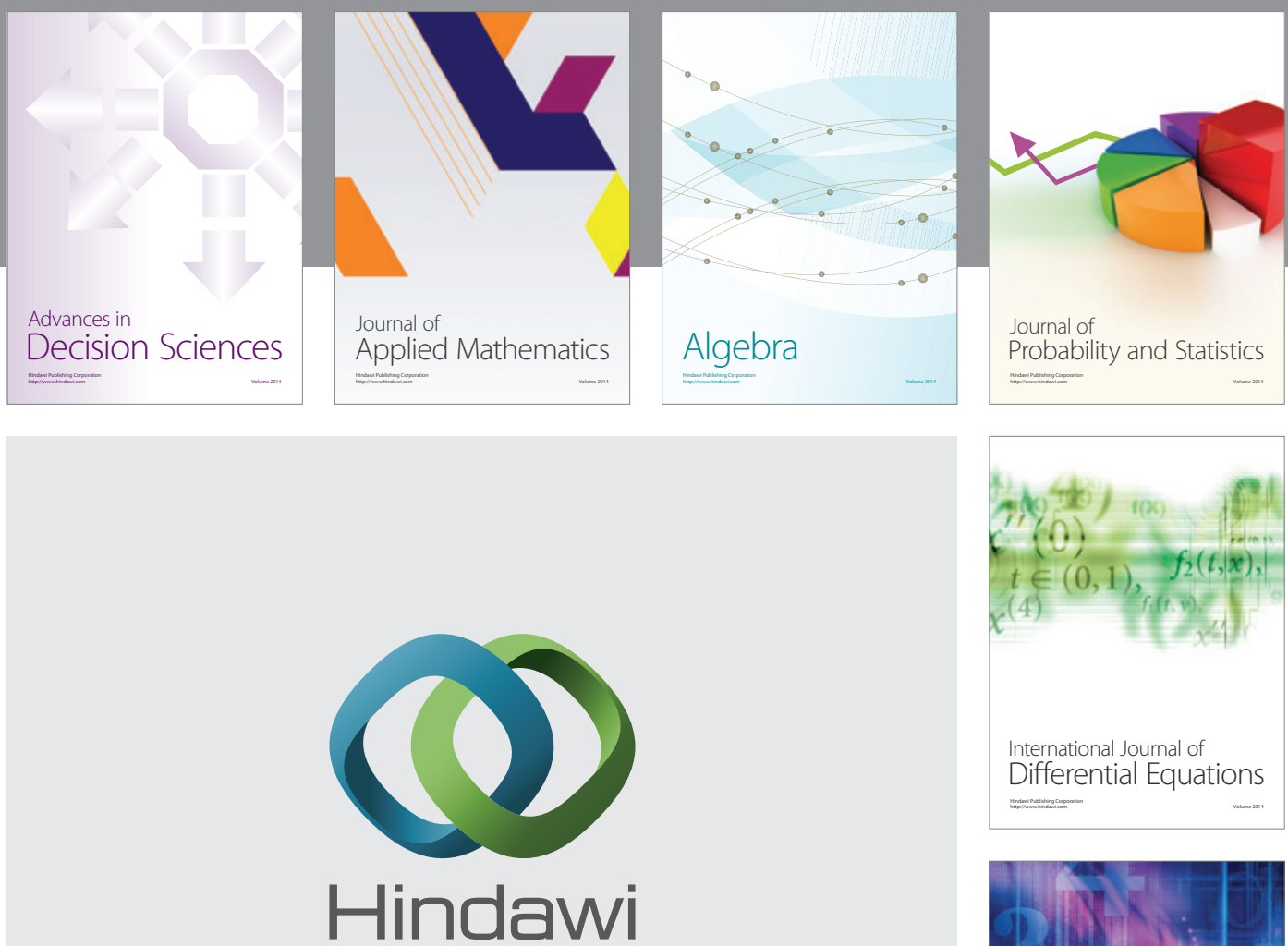

Submit your manuscripts at http://www.hindawi.com
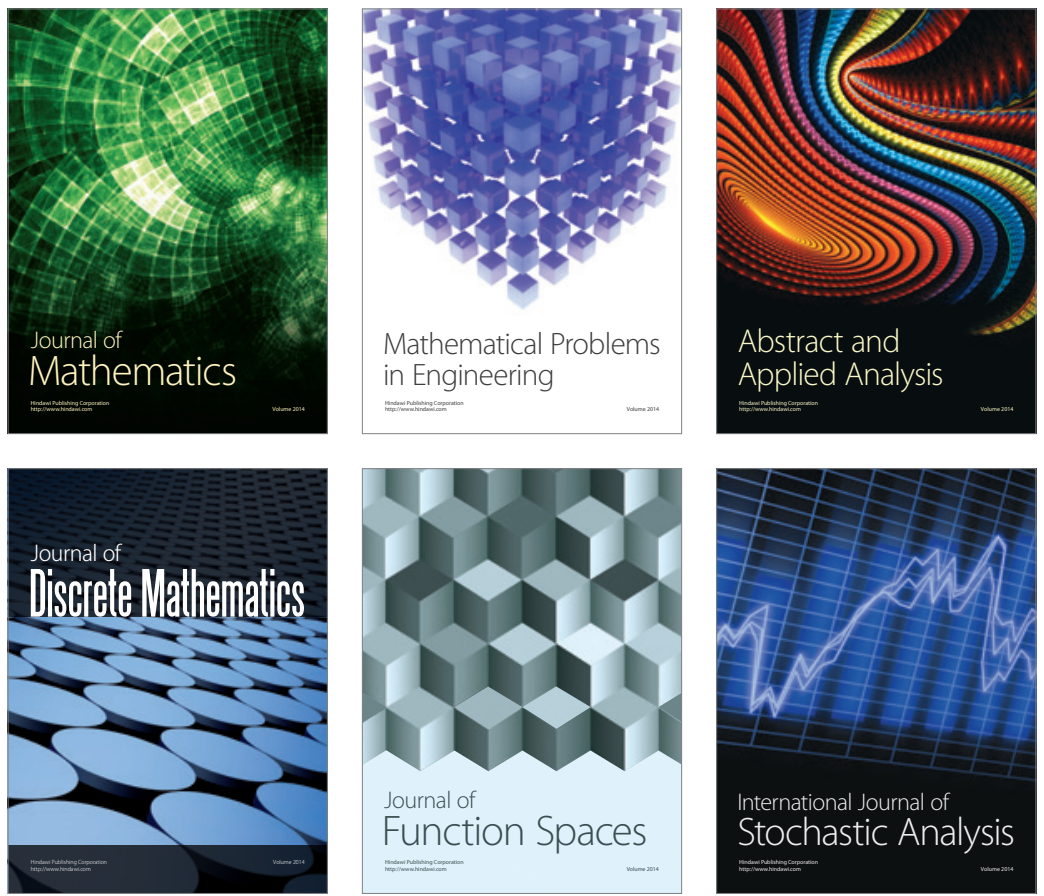

Journal of

Function Spaces

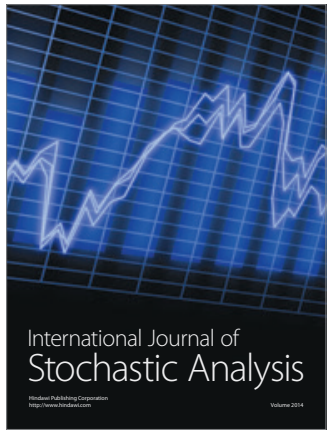

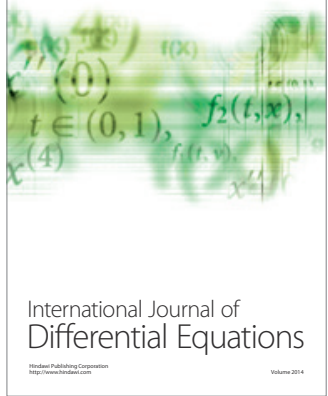
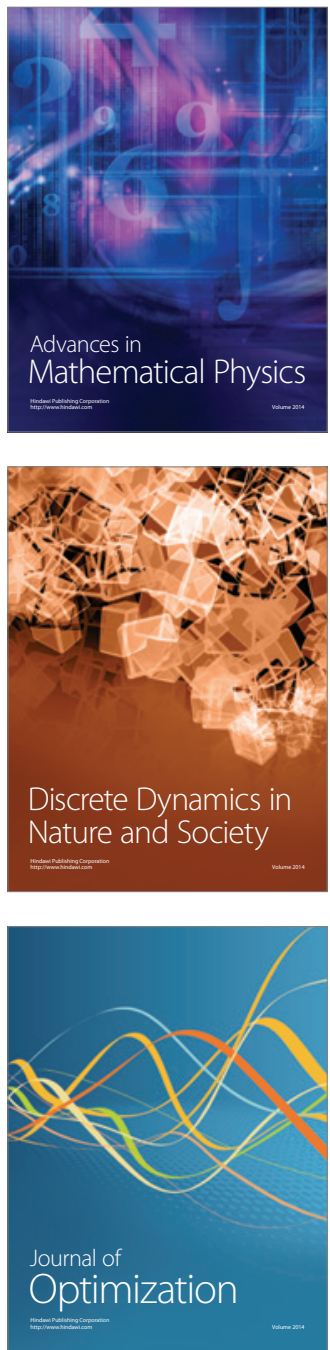